\title{
Nuclear medicine and immunotherapy: many questions but not many answers yet
}

\author{
Salvatore Annunziata ${ }^{1} \cdot$ Alessandro Giordano $^{1,2}$ \\ Received: 19 October 2018 / Accepted: 21 December 2018 / Published online: 23 January 2019 \\ (c) Italian Association of Nuclear Medicine and Molecular Imaging 2019
}

Keywords Imaging $\cdot$ Immunotherapy $\cdot$ PET $\cdot$ Monoclonal antibodies $\cdot$ Personalised medicine

In recent years, several types of immuno-therapies have been developed for cancer treatment, deeply changing paradigms and needs of clinicians, radiologists and Nuclear physicians. Research provided promising results through several approaches: "immune checkpoint inhibitors", "therapeutic antibodies", "therapeutic vaccines", "immune-modulating agents". Certainly, PD-1/PD-L1 immune checkpoint inhibitors are raising the highest interest due to demonstration of their efficacy in the treatment of relapsed/refractory lymphomas, as well as in other tumors like melanoma and lung cancer [1-3]. This new era of personalized medicine for patients with cancer opens new challenges for Nuclear Medicine and molecular imaging: to deeply understand new clinicians' needs about treatment evaluation imaging and to possibly propose new imaging and therapeutic tools by radiolabeling monoclonal antibodies.

What are "immune checkpoint inhibitors"? In normal conditions, autoimmunity is prevented by the interaction of PD-1 receptors (PD-L1 and PD-L2), expressed by normal cells, and PD-1, expressed by T lymphocytes. These molecules are called checkpoint proteins. Some tumors have been shown to be capable of a rapid proliferation due to an overexpression of PD-1 receptors, thus blocking immune response by T lymphocytes [4]. Drugs capable of inhibiting the interaction between the checkpoint proteins PD-1 and PD-L1 have been demonstrated to allow the immune system to strongly oppose to the tumor growth. These drugs are called "immune checkpoint inhibitors": among them,

Salvatore Annunziata

salvatoreannunziata@live.it

1 Institute of Nuclear Medicine, Università Cattolica del Sacro Cuore, Largo Agostino Gemelli 8, 00168 Rome, Italy

2 UOC Medicina Nucleare, Dipartimento Diagnostica per Immagini, Radioterapia Oncologica ed Ematologia, Fondazione Policlinico A. Gemelli IRCCS, Rome, Italy
Nivolumab and Pembrolizumab are anti-PD-1/PD-L1 agents that have been introduced into clinical practice in Europe for the treatment of several cancer histotypes with great efficacy [1-4].

Such a new therapeutic approach soon raised key questions: is imaging adequate to assess the response to these treatments? How can it predict response to treatment? Has FDG-PET the same priceless role as in the evaluation of response to standard chemotherapy? Are new biomarkers required? Can we radiolabel checkpoint inhibitors to non-invasively map PD-L1 expression? In recent issues of Clinical and Translational Imaging, some interesting reviews have been published, respectively, discussing lung cancer and lymphoma imaging in the era of immunotherapy and radiopharmacology of PD-L1 in cancer [1-3].

In the paper by Ciarmiello et al., the role of imaging in non-small cell lung cancer (NSCLC) immunotherapy has been discussed [1]. Immunotherapy deeply changed treatment management of NSCLC patients, since Nivolumab and pembrolizumab have been approved by FDA and EMA as second-line therapy in advanced NSCLC. About immunotherapy imaging, immune-related response criteria (irRC) and immune-related RECIST (irRECIST) have been developed to update conventional diagnostic criteria, taking into account different time-points of treatment evaluation and apparent increase of the tumor lesions (so-called "pseudoprogression"). Despite the pitfalls of pseudoprogression and the peculiar patterns of immunotherapy response, 18F-FDG PET-CT seems to remain the primary imaging modality for the evaluation of tumor response also to immunomodulatory agents in NSCLC patients. According to recent evidence, an objective response to PD1/PD-L1 inhibitors was reported to occur approximately 2 months after the beginning of therapy [1]. However, a second follow-up scan at 4-8 weeks seems to be recommended to confirm or not disease progression. The enhancement of FDG uptake in other organs could be 
considered for the evaluation of immune-related adverse effects. About patients' selection for immunotherapy, PD-L1 status could be useful, since high levels of PD-L1 were associated with improved survival, but its role is not clear for different histology and immunotherapy regimens [1].

In another review by Kirienko et al., evidence on the role of imaging in Hodgkin Lymphomas was reported, with focus on PET/CT and therapy with PD-1/PD-L1 immune checkpoint inhibitors, summarizing potentials and limitations [2]. In fact, PET/CT clearly improved the management of lymphomas both in staging and treatment evaluation settings in the last 15 years, overcoming several limitations of morphologic imaging as CT. Visual scores have been implemented for treatment evaluation as the Deauville Score and the more recent Lugano/Lyric criteria. Moreover, new PET-derived semi-quantitative and radiomic parameters have been recently proposed as biomarkers to stratify different risk populations and improve their treatment management [5]. Nevertheless, some issues remain in the routine use of FDG$\mathrm{PET} / \mathrm{CT}$ in response to immunotherapy evaluation, mainly consisting in the identification of effective biomarkers for the selection of treatable patients and standardized response criteria for PET imaging [6]. In fact, hematological biomarkers as PD-L1 expression levels still are sub-optimal to individuate patients eligible for immunotherapy. With this aim, new circulating nucleic acids as cDNA have been recently proposed and compared with PET imaging on the diagnostic value point of view [7]. On the other hand, the role of FDGPET/CT in the evaluation of response to immunotherapy is far from being recognized by international guidelines. The use of an increasing number of response evaluation criteria needs to be standardized in clinical practice and research protocols, especially in comparison with actual standard morphological imaging criteria and in the context of new hybrid imaging (e.g., PET/MR). The impact of the so-called "pseudo-progression" and the worrying introduction of the "indeterminate response" have to be clarified, to avoid chills down the spine of the experienced nuclear physicians who remember the case of PIOPED criteria for lung scan. Last but not least, a new concept of short-term FDG-PET/CT follow-up emerged in immuno-therapy monitoring, mining old paradigms that excluded the use of FDG in the oncological follow-up. Kirienko et al. review's conclusion suggests that a potential breakthrough solution could be the use of the so-called immunoPET or immunoSPECT scans, using radiolabeled anti-PD-1/PD-L1 complexes capable of scintigraphically visualizing in vivo immune checkpoint expression and its variation in time [2].

The review by Vaz et al. starts exactly from here. The authors reviewed published data about imaging of PD-L1 expression by radiotracers that incorporated single-photon or positron-emitting isotopes, including in vitro researches, pre-clinical studies and a few pilot clinical trials [3]. After the high impact and diffusion of immunotherapy among oncological patients, first hypotheses and pre-clinical studies have been proposed on the use of radiolabeled antibodies for both imaging and radioimmunotherapy, with the aim to explore new theranostic tools in oncology. Several radioisotopes have been proposed for both SPECT and PET applications, mostly $89 \mathrm{Zr}, 18 \mathrm{~F}$ and $64 \mathrm{Cu}$ (so-called immunoPET) [8]. Since PD-L1 targeted therapy is one of the most frequently used and effective immunotherapies in several tumors (e.g., lymphoma, melanoma, lung cancer), first studies about molecular imaging and therapy proposed to radiolabel anti-PD-L1 antibodies. In the review by Vaz et al., radiolabeled antibodies showed the potentiality to mark PD-L1 expression, with their possible use as an imaging tool before and after immunotherapy, to impact on cancer management and even for their further use as radioimmunotherapy [3]. The use of immuno-PET as a biomarker could be of great help for the individuation of eligible oncological patients for immunotherapy, as already happens for somatostatin receptor ligands or new PSMA ligands and patients with neuroendocrine tumors and prostate cancer. In other words, radiolabeled antibodies could be the "Nuclear Medicine answer" in the era of personalized and precision medicine, when immunotherapy is quickly spreading worldwide as a curative and maintenance oncological therapy. Nevertheless, the use of PDL1 radiotracers in clinical practice still seems far and difficult. Until now, only pre-clinical studies have been published and few clinical trials started [3]. Clinical, dosimetric and economic issues emerged on the road of routine use of these new radiotracers in clinical practice. As Nuclear physicians, our knowledge about biology and pharmacology of monoclonal antibodies should be further implemented, to better understand potentialities and limitations of radio-labeling. As well as immunotherapy, radioimmunotherapy can result in unwanted activation of autoimmunity, causing a wide range of adverse effects. Safety, dosimetric and cost-efficacy analyses in humans are still lacking and should be further studied to propose this approach in the clinical scenario [3, 9]. Starting from past experiences with radioimmunotherapy, the Nuclear Medicine community should go deeper in clinical and research potentialities of new PDL1 radiopharmaceuticals. Cooperation with clinicians, private and public institutions in Italy and Europe is needed to facilitate the routine use of new radiopharmaceuticals and really take part in the clinical management of many oncological patients.

Another desirable cooperation might also include radiologists. Applications of radiomics to contrast-enhanced CT images have recently allowed to identify an imaging predictor of immune phenotype of tumors (called "radiomic signature") capable to infer clinical outcomes for patients treated with PD-1/PD-L1 immune checkpoint inhibitors. These information might be considered complementary to those 
derived from radiopharmaceuticals; the widespread availability of PET-CT tomographs would theoretically facilitate such an hybrid approach [10].

Finally, the reviews discussed here offer wide possibilities and suggestions for further researches [1-3]. In fact, the following uncertainties still remain: (1) are more robust evidence needed to apply new diagnostic criteria to immunotherapy evaluation? (2) can new diagnostic criteria be applied to different cancer histotypes and immunotherapy regimens? (3) will guidelines and scientific societies endorse these criteria? Moreover, we believe that the assessment of the incremental economic impact and incremental clinical benefits of these therapeutic approaches using immune checkpoint inhibitors is necessary, to justify the costs required and the saves provided by the development and use of new immuno-radiopharmaceuticals. Since not every patient who receives immunotherapy will respond to the treatment, the identification of proper biomarkers is necessary to predict response to immunotherapy and improve patients' selection. Eventually, if able to give answers to these questions, Nuclear Medicine and molecular imaging will play a central role in this field in the next years.

Author contributions SA: Literature search and review, Manuscript writing. AG: Content planning, manuscript writing and editing.

\section{Compliance with ethical standards}

Conflict of interest SA and AG declare that they have no conflict of interest.

Human and animal rights statement This article does not contain any studies with human participants performed by any of the authors.

\section{References}

1. Ciarmiello A, Fonti R, Giovacchini G et al (2018) Imaging of immunotherapy response in non-small cell lung cancer: challenges and perspectives. Clin Transl Imaging 6:483

2. Kirienko M, Sollini M, Chiti A (2018) Hodgkin lymphoma and imaging in the era of anti-PD-1/PD-L1 therapy. Clin Transl Imaging 6:417

3. Vaz SC, Capacho AS, Oliveira FP et al (2018) Radiopharmacology and molecular imaging of PD-L1 expression in cancer. Clin Transl Imaging 6:429

4. Gordon SR, Maute RL, Dulken BW et al (2017) PD-1 expression by tumour-associated macrophages inhibits phagocytosis and tumour immunity. Nature 545:495-499

5. Annunziata S, Cuccaro A, Tisi MC et al (2018) FDG-PET/CT at the end of immuno-chemotherapy in follicular lymphoma: the prognostic role of the ratio between target lesion and liver SUV(max) (rPET). Ann Nucl Med 32(5):372-377

6. Rossi S, Toschi L, Castello A et al (2017) Clinical characteristics of patient selection and imaging predictors of outcome in solid tumors treated with checkpoint-inhibitors. Eur J Nucl Med Mol Imaging. 44(13):2310-2325

7. Spina V, Bruscaggin A, Cuccaro A et al (2018) Circulating tumor DNA reveals genetics, clonal evolution, and residual disease in classical Hodgkin lymphoma. Blood 131(22):2413-2425

8. Mayer AT, Natarajan A, Gordon SR et al (2017) Practical immuno-PET radiotracer design considerations for human immune checkpoint imaging. J Nucl Med 58(4):538-546

9. Josefsson A, Nedrow J, Park S et al (2016) Dosimetric analysis of Actinium-225 labeled anti-PD-L1 alpha particle radioimmunotherapy of melanoma cancer in an immunocompetent mouse model. J Nucl Med 57(Supplement 2):635

10. Sun R, Limkin EJ, Vakalopoulou M et al (2018) A radiomics approach to assess tumour-infiltrating CD8 cells and response to anti-PD-1 or anti-PD-L1 immunotherapy: an imaging biomarker, retrospective multicohort study. Lancet Oncol. 19(9):1180-1191 